\title{
Ultrafast lattice dynamics in lead selenide quantum dot
}

\author{
Xuan Wang ${ }^{1, *}$, Matthew Gorfien ${ }^{2}$, and Jianming $\mathrm{Cao}^{2,3, * *}$ \\ ${ }^{1}$ Institute of Physics, Chinese Academy of Sciences, P.O. Box 603, Beijing, 100190, China \\ ${ }^{2}$ Physics Department and National High Magnetic Field Laboratory, Florida State University, Tallahas- \\ see, Florida, 32310, USA \\ ${ }^{3}$ Center for Ultrafast Science and Technology, Key Laboratory for Laser Plasmas (Ministry of Ed- \\ ucation), School of Physics and Astronomy, Collaborative Innovation Center of IFSA (CICIFSA), \\ Shanghai Jiao Tong University, Shanghai, 200240, China
}

\begin{abstract}
We monitored the femtosecond-laser-induced lattice dynamics in $\mathrm{PbSe}$ quantum dots by ultrafast electron diffraction. The electron-phonon coupling didn't show phonon bottleneck. And lattice dilation exhibited unusual features. Heat transport to the substrate deviated significantly from Fourier's Law.
\end{abstract}

\section{Introduction}

Lead selenide $(\mathrm{PbSe})$ quantum dots $(\mathrm{QD})$ are photoactive in the near infrared region with very high photoluminescence quantum yields. Electron and hole Bohr radii in PbSe QDs are so large (about $23 \mathrm{~nm}$ ) that both carriers experience very strong confinement in sub-10 nm particles [1]. The strong confinement makes the electronic density of states very discrete with the energy difference between adjacent electronic states exceeding several optical phonon energies. It has been predicted that carrier-phonon relaxation rate is significantly reduced (phonon bottleneck). However, the experimental evidences for phonon bottleneck effect have been controversial with both pros and cons [2-4], and several theoretical models bypassing phonon bottleneck are proposed $[2,5]$. In this paper, we demonstrated a method of probing the electron-phonon coupling in QDs through directly monitoring the lattice system with ultrafast electron diffraction (UED). In particular, we traced the lattice temperature, its dilation, and the heat transport from QDs to the substrate.

\section{Methods}

The experiment was conducted on the UED instrument using $60 \mathrm{keV}$ electron with an overall temporal resolution less than $500 \mathrm{fs}$. [6] The QDs with average size and size distribution $5.8 \mathrm{~nm}$ and $0.2 \mathrm{~nm}$ (standard deviation) respectively, were synthesized and dip-coated onto $50 \mathrm{~nm}$ thick $\mathrm{Si}_{3} \mathrm{~N}_{4}$ membranes . [7] Static measurements were conducted to determine the linear expansion coefficient and the Debye-Waller factor (DWF). [8] The DWF is then used to covert the intensity change of diffraction peaks into lattice temperature change in an UED experiment. In the experiment, the sample base temperature was $146 \mathrm{~K}$ and lattice dynamics was initiated by $800 \mathrm{~nm}, 50$ fs laser pulses at $\sim 5 \mu \mathrm{J} \mathrm{mm}^{-2}$.

\footnotetext{
*e-mail: xw@iphy.ac.cn

**e-mail: jcao@magnet.fsu.edu
} 


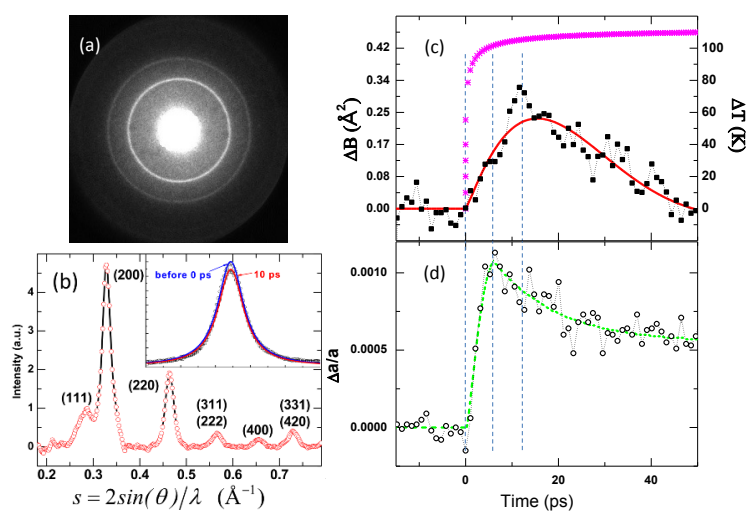

Figure 1. (a) A typical DP of PbSe QDs and (b) the corresponding intensity profile after background substraction. Inset: the intensity profile of $(2,2,0)$ peak at $0 \mathrm{ps}$ (open circle) and $10 \mathrm{ps}$ (open square) after time zero, respectively. The solid curves are fittings by a Lorentz function. (c) The change of DWF (solid square) as a function of time. The solid line is a model fitting. The asterisk line is a simulation of the evolution of carriers' excessive energy due to Auger relaxation. (d) Relative lattice spacing change (open circle) as a function of time. The dashed line is for guiding viewer's eyes.

\section{Results and discussion}

As shown in Fig.1, the lattice temperature (extracted from DWF) and lattice spacing change (extracted from the position of the Bragg peaks in Fig.1(b)) were not in pace, reflecting unusual aspects of lattice dynamics. Specifically, the lattice temperature rose in about $10 \mathrm{ps}$ and then fell back to the original value in about $40 \mathrm{ps;}$ while the lattice spacing showed an initial 6 ps rise faster than lattice temperature, and didn't return back to the original value. The QD dynamics is set by the electron-electron scattering, carrier-phonon scattering, heat flow from QDs to the substrate. After absorbing $1.55 \mathrm{eV}$ photons, the electrons are excited into the conduction band with an excessive energy $E_{e x} \approx 0.75 \mathrm{eV}$. These carriers redistribute their energies among themselves by various carrier-carrier scattering process. Particularly, via Auger scattering [9], a portion of the electrons and holes recombine while transferring their energy to the remaining ones. Meanwhile, the carriers relax their energy to the lattice system by carrier-phonon scattering. The above process is modeled by [8]

$$
\begin{aligned}
\frac{d E_{e x}}{d t} & =\frac{A E_{g} V n_{0}^{3}}{\left(1+2 A n_{0}^{2} t\right)^{1.5}}-\frac{E_{e x}}{\tau}+S(t) \\
C_{p} \frac{d T_{l}}{d t} & =\frac{E_{e x}(t)}{\tau}+q \\
q & =-\tau_{R} \frac{d q}{d t}-h\left(T_{l}-T_{s}\right)
\end{aligned}
$$

where $\mathrm{V}$ is the average volume of the QDs, $\tau$ is the time constant that characterizes carrierphonon coupling, and $S(t)$ is the laser pumping term. $n_{0}$ is the initial density of excited carriers. $C_{p}$ is the lattice heat capacity [10], and $T_{l}$ is the lattice temperature. $q$ represents the energy flow between the QD and the substrate. The first term on the right hand of Eq.1 represents the Auger relaxation process with the Auger constant $A=5 \times 10^{-42} \mathrm{~m}^{6} \mathrm{~s}^{-1}$ [11]. Eq.3 is the Cattaneo equation [12] with $\tau_{R}$ as the retarded time constant and $h$ as the product of thermal contact conductance and contact area. We define $\tau_{\text {heat }}=C_{p} / h$ as the time constant of heat transport between the QD and the substrate. We take $\tau_{R}, \tau$, and $\tau_{\text {heat }}$ as fitting parameters, 
and the evolution of $E_{e x}$ due to Auger relaxation alone is also plotted. We obtained $\tau \approx 10 \mathrm{ps,}$ no longer than the bulk value, indicating no phonon bottleneck. Regarding the heat transport to substrate, $\tau_{R}=10 \mathrm{ps}$ was very critical for the data fitting, which implies that under a non-thermal-far-from-equilibrium condition established in QDs with $T_{e}$ does not equal $T_{l}$, the Fourier's Law doesn't hold any more. [13]

The transient lattice spacing change in Fig.1 (d) also raised some interesting points. The lattice dilation can be driven by carriers and/or phonons and is sometimes accompanied by a coherent vibration at the beginning [14]. The total stress can be expressed by [15]

$$
\sigma=\sigma_{T E}+\sigma_{D P}+\sigma_{o t h e r}
$$

where $\sigma_{T E}$ is the phonon stress due to thermal elasticity, $\sigma_{D P}$ is the stress from carriers due to the deformation potential, and $\sigma_{\text {other }}$ accounts for all other contributions. The lattice expansion is ahead of temperature indicating the significant contribution of $\sigma_{D P}$ and $\sigma_{\text {other }}$. But $\sigma_{D P}$ is compressive when all carriers relax to the bottom of the conduction band. After about $40 \mathrm{ps}$ when lattice temperature returns back, there is still about $0.05 \%$ dilation remaining. So $\sigma_{\text {other }}$ must be tensile. We speculate that QD charging by pumping may expand QDs. [16] This work is supported by National Science Foundation of China (Grant No.11774409), the National Science Foundation of USA (Grant No.1207252), National Science Foundation Cooperative Agreement No. DMR-1157490 and the State of Florida.

\section{References}

[1] F.W. Wise, Accounts of Chemical Research 33, 773 (2000)

[2] S. Kilina, B.F. Habenicht, Excitonic and Vibrational Dynamics in Nanotechnology (Pan Stanford Publishing, 2009)

[3] B.L. Wehrenberg, C. Wang, P. Guyot-Sionnest, The Journal of Physical Chemistry B 106, 10634 (2002)

[4] J.M. Harbold, H. Du, T.D. Krauss, K.S. Cho, C.B. Murray, F.W. Wise, Physical Review B 72, 195312 (2005)

[5] R.D. Schaller, J.M. Pietryga, S.V. Goupalov, M.A. Petruska, S.A. Ivanov, V.I. Klimov, Physical review letters 95, 196401 (2005)

[6] X. Wang, S. Nie, J. Li, R. Clinite, M. Wartenbe, M. Martin, W. Liang, J. Cao, Applied Physics Letters 92, 121918 (2008)

[7] A. Wolcott, V. Doyeux, C.A. Nelson, R. Gearba, K.W. Lei, K.G. Yager, A.D. Dolocan, K. Williams, D. Nguyen, X.Y. Zhu, The Journal of Physical Chemistry Letters 2, 795 (2011)

[8] X. Wang, H. Rahmani, J. Zhou, M. Gorfien, J. Mendez Plaskus, D. Li, R. Voss, C.A. Nelson, K. Wai Lei, A. Wolcott et al., Applied Physics Letters 109, 153105 (2016)

[9] V.I. Klimov, A. Mikhailovsky, D. McBranch, C. Leatherdale, M.G. Bawendi, Science 287, 1011 (2000)

[10] D. Parkinson, J. Quarrington, Proceedings of the Physical Society. Section A 67, 569 (1954)

[11] V.I. Klimov, The Journal of Physical Chemistry B 110, 16827 (2006)

[12] Z. Zhang, Nano/Microscale Heat Transfer (McGraw-Hill: New York, 2007)

[13] G.M. Vanacore, J. Hu, W. Liang, S. Bietti, S. Sanguinetti, A.H. Zewail, Nano letters 14, 6148 (2014)

[14] H. Park, X. Wang, S. Nie, R. Clinite, J. Cao, Physical Review B 72, 100301 (2005)

[15] P. Ruello, V.E. Gusev, Ultrasonics 56, 21 (2015)

[16] T.D. Krauss, F.W. Wise, Physical review letters 79, 5102 (1997) 